\title{
Avaliação comparativa da adaptação de lentes de contato rígidas gás-permeáveis Monocurvas e Bicurvas (desenho Soper-McGuire) em pacientes portadores de ceratocone: um estudo prospectivo e randomizado
}

\author{
Fitting Monocurve and Bicurve (Soper-McGuire design) rigid gas-permeable contact \\ lenses in keratoconus patients: a prospective randomized comparative clinical trial
}

Cleusa Coral-Ghanem ${ }^{1}$

Milton Ruiz Alves²

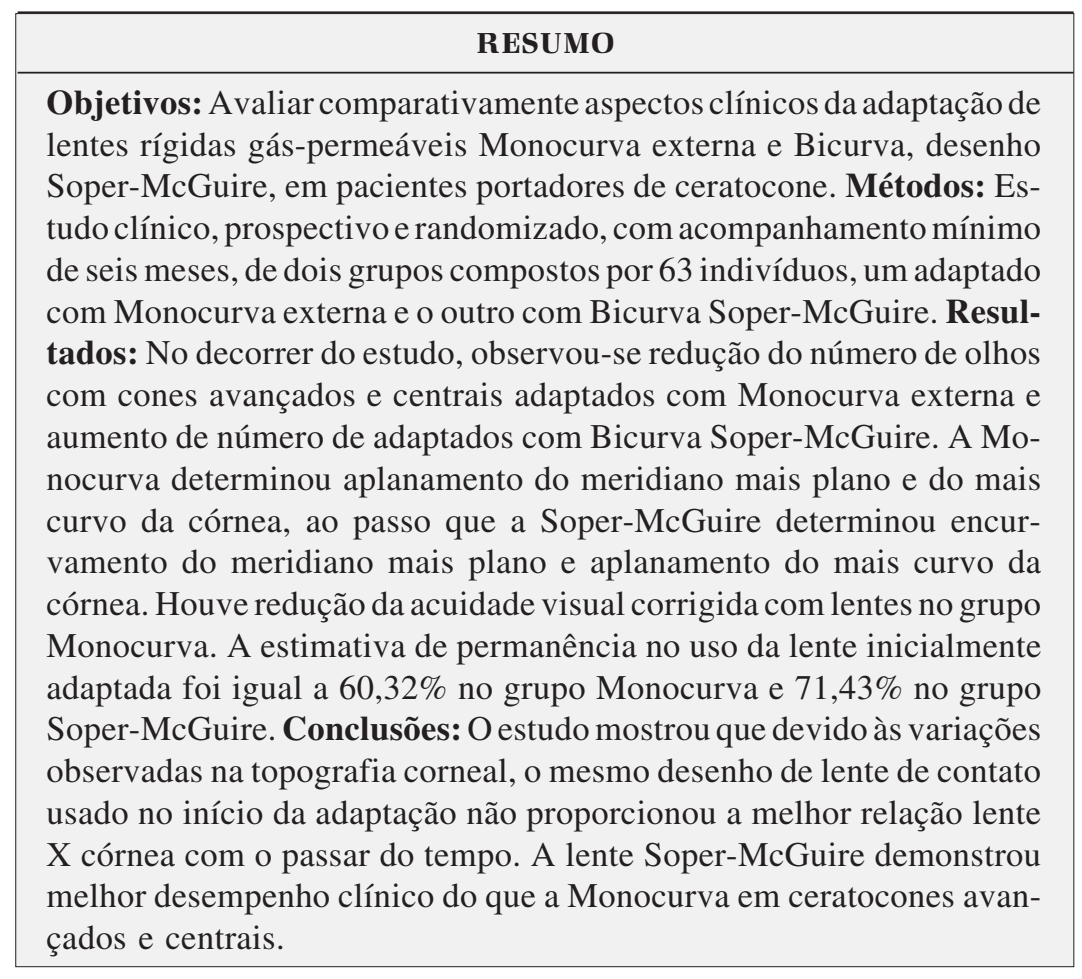

Descritores: Ceratocone; Lentes de contato; Evolução clínica; Topografia da córnea; Acuidade visual

\section{INTRODUÇÃ̃O}

O ceratocone tem sido considerado uma ectasia corneal não inflamatória, progressiva, assimétrica, na qual a porção central ou paracentral da córnea assume forma cônica, permanecendo a periférica relativamente normal. Com a progressão da doença ocorre protrusão apical, astigmatismo irregular, afilamento do estroma corneal, formação de cicatrizes corneais e importante baixa visual ${ }^{(1-2)}$.

A adaptação de lentes de contato (LC) rígidas é o tratamento padrão para a recuperação visual do portador de ceratocone, pois elas proporcionam uma superfície regular em substituição a da córnea distorcida(3), 
retardando ou evitando o encaminhamento do paciente à cirurgia $^{(1,3-7)}$. Em torno de $90 \%$ dos pacientes com ceratocone optam pelas $\mathrm{LC}^{(8-12)}$.

Uma análise multicêntrica sobre a conduta clínica em ceratocone mostrou que de $74 \%$ dos portadores desta doença, que poderiam ter sido submetidos à cirurgia, 84\% ficaram bem adaptados com LC ${ }^{(13)}$. Estudos mostraram que em torno de $80 \%$ dos pacientes com ceratocone, adaptados com LC, alcançaram acuidade visual (AV) de 20/40 ou melhor ${ }^{(8,11,13-16)}$.

Embora as LC mais utilizadas nas últimas duas décadas sejam as rígidas gás-permeáveis (RGP) com desenho monocurvo, esférico ou asférico, e as com desenho bicurvo tipo Soper ${ }^{(11,16-19)}$, em levantamento bibliográfico extenso não foi possível encontrar publicações que comparem o desempenho clínico entre estes dois tipos de desenho.

Considerando a importância da adaptação de LC para a recuperação visual de portadores de ceratocone, o objetivo do presente estudo foi o de comparar o desempenho clínico das LC RGP Monocurvas e Bicurvas nos diferentes graus de evolução desta doença.

\section{MÉTODOS}

Realizou-se estudo clínico, prospectivo e randomizado para avaliar aspectos clínicos associados à adaptação e ao desempenho de dois tipos de LC RGP, Monocurvas e Bicurvas tipo Soper (desenho Soper-McGuire - Optolentes-RS ${ }^{\circledR}$ ), em pacientes portadores de ceratocone que procuraram o setor de Lentes de Contato do Hospital de Olhos Sadalla Amin Ghanem, entre $1^{\circ}$ de julho de 2000 e 28 de fevereiro de 2002.

\section{Formação dos grupos}

Numa primeira fase foram examinados consecutivamente 397 pacientes portadores de ceratocone, usuários ou não de LC, com indicação para o uso de LC. Nesta etapa foram excluídos 187 pacientes pelas razões: visão satisfatória sem correção óptica ou com o uso de óculos (20/30 ou melhor); meridiano mais plano da córnea com poder dióptrico inferior a 45,00 dioptrias (D); usuários de outros tipos de LC; pessoas que não dispunham de tempo para as revisões de seguimento; casos com a indicação para transplante de córnea; e aqueles que já haviam desistido do uso de LC. Os outros 210 pacientes foram submetidos a testes de adaptação com LC Monocurvas e Bicurvas tipo Soper (desenho Soper-McGuire), com o objetivo de selecionar aqueles que pudessem ser adaptados com os dois tipos de LC. Foi solicitado aos usuários de LC que suspendessem o uso por pelo menos 15 dias. Ao final desta etapa, foram excluídos 84 pacientes que não cumpriram os critérios de elegibilidade. Para compor um grupo controle, com o objetivo de analisar a validade externa do estudo, foram selecionados por sorteio 61 desses pacientes excluídos, dos quais obtiveram-se dados demográficos e clínicos levantados pelo exame oftálmico e pelo teste com as LC. Depois dessa triagem, a população do estudo ficou composta por 126 pacientes que concordaram em participar do estudo. Assinado o Termo de Consentimento, foram admitidos para a etapa seguinte e randomizados por sorteio para comporem dois grupos de 63 pacientes: Grupo Monocurva (ambos os olhos adaptados com LC RGP Monocurva externa) e Grupo Bicurva (ambos os olhos adaptados com LC RGP Bicurvas Soper-McGuire). Cada paciente teve um olho escolhido por sorteio para a avaliação do desempenho clínico da LC. As variáveis selecionadas foram classificadas da seguinte maneira: grau do ceratocone pelos valores ceratométricos ${ }^{(6)}$; localização do ápice do cone identificado pelo mapa ceratométrico do Orbscan $\mathrm{II}^{\mathrm{TM}}$ : central (dentro de $3 \mathrm{~mm}$ ); paracentral (entre 3 e $5 \mathrm{~mm}$ ) e periférico (além dos $5 \mathrm{~mm}$ centrais); meridiano mais plano da córnea: Kmin; meridiano mais curvo da córnea: Kmax; poder dióptrico médio da córnea: $\mathrm{Km}=(\mathrm{Kmin}+\mathrm{Kmax}) / 2$; toricidade da córnea: Kmax - Kmin; relação LC X córnea pela análise do padrão fluoresceínico; AV para a distância com a melhor correção óptica, expressa em LogMar; retenção de pacientes e descontinuidade do estudo. Os critérios de inclusão para a amostra foram: pessoas de ambos os sexos portadoras de ceratocone, usuários ou não de LC, com indicação para o uso, por apresentarem AV bilateral, corrigida com óculos, igual ou inferior a 20/30; meridiano mais plano da córnea com poder dióptrico superior a 45,00 D; cones moderados, avançados ou muito avançados que pudessem ser adaptados tanto com LC Monocurva quanto com LC Bicurva, tipo Soper-McGuire; usuários de LC que estavam, pelo menos, há 15 dias sem usá-las, ou que concordaram em suspendê-las por este período; assinatura do Termo de Consentimento Livre e Escalarecido. Os critérios de exclusão foram: alterações oculares, além do ceratocone, que reduzissem a AV; alterações conjuntivais e palpebrais que pudessem diminuir o conforto (por exemplo: conjuntivite papilar gigante e blefarite); cones periféricos; córneas que não puderam ser adaptadas com os dois tipos de LC em estudo; desconforto importante no teste de adaptação com as LC selecionadas; desacordo com os critérios de inclusão e randomização.

\section{Materiais}

Os dois tipos de LC foram fabricados pela Optolentes ${ }^{\circledR}$ (Porto Alegre, RS) com o material Boston EO Envision (enflufocon $\mathrm{B}$, acrilato de fluorosiloxane) que possui entrelaçamento molecular AERCOR ${ }^{\circledR}$. Esse material apresenta boa estabilidade e transmissibilidade ao oxigênio $\left(\mathrm{O}_{2}\right)(\mathrm{Dk}=82$, pelo método "gas to gas" e $\mathrm{Dk}=58$ pelo ISO/Fatt). Seu conteúdo de silicone é baixo (entre 5 e 7\%), permitindo excelente umectação e resistência a depósitos (ângulo de umectação de $49^{\circ}$ ). O índice de dureza do material pelo método Rockwell é 114 e pelo Shore-D é de 83. O desenho da LC RGP Monocurva externa apresenta sua face anterior como uma curva contínua e a posterior com três ou mais curvas.

O desenho da LC RGP Bicurva, Soper-McGuire é derivado do desenvolvido por Soper ${ }^{(20)}$, acrescido de curvas nas zonas de transição entre a curva base (CB) da zona óptica (ZO) e da zona intermediária e entre a $\mathrm{CB}$ da zona intermediária e a 
periferia. Essas modificações deixaram seu desenho semelhante ao da LC McGuire (Soper modificada fabricada nos Estados Unidos) e, por isso, foi denominada Soper-McGuire. Esta LC apresenta duas $\mathrm{CB}$ na face posterior. A central, mais apertada (de 47,00 a 70,00 D), é projetada para adaptar sobre o cone e a secundária, mais plana (de 45,00 a 55,00 D), para apoiar na córnea mediana periférica. Sua espessura central é de $0,10 \mathrm{~mm}$ ou menos e a ZO pode ter diferentes tamanhos. A de maior tamanho é utilizada para cones de diâmetros $(\varnothing)$ grandes e a de menor para cones centrais tipo "nipple". A CB secundária também pode ser solicitada com outras medidas, de acordo com a forma e o grau do cone.

\section{Procedimentos e técnicas}

Nas avaliações programadas e nos retornos não agendados, os pacientes incluídos no estudo foram submetidos aos seguintes exames: anamnese, exame ocular externo, refratometria dinâmica ("Topcon Compuvision CV 2500", com projetor "Mirror Chart CM2"), medida da AV corrigida com as LC, biomicroscopia do segmento anterior e análise do padrão fluoresceínico com lâmpada de fenda "Topcon-modelo SRL", fundoscopia direta, paquimetria e topografia de curvatura e elevação pelo "Orbscan II ${ }^{\mathrm{TM}}$ - Bausch\&Lomb Surgical, Rochester, NY”.

Para a adaptação das LC RGP Monocurvas e Bicurvas Soper-McGuire foi utilizada uma das seguintes técnicas: livramento apical; toque apical e três pontos de toque (um central e dois periféricos). Sempre que possível, iniciou-se a adaptação com a técnica de livramento apical.

\section{Livramento apical}

Neste método, a primeira LC de teste teve o raio da CB igual ao valor da curvatura ceratométrica mais apertada. Em presença de bolha, aplanou-se a CB da LC até encontrar a CB mais plana que não demonstrasse toque apical e que apresentasse livramento periférico maior que $180^{\circ}$ para não impedir o fluxo lacrimal. Para evitar a adesão da LC, algumas vezes foi realizada a abertura das curvas periféricas, no consultório. $\mathrm{O}$ $\varnothing$ inicial da Monocurva foi $8,6 \mathrm{~mm}$ e da Bicurva, $9 \mathrm{~mm}$.

\section{Toque apical}

Nesta técnica, necessária para os casos de descentralização da LC e/ou de $\varnothing$ pupilar grande, foi utilizada LC com $\varnothing$ grande e CB relacionada com o valor da curva ceratométrica mais plana. O padrão fluoresceínico mostrou, geralmente, moderado toque no ápice e na meia periferia e livramento periférico amplo. Foi evitado, sempre que possível, LC com toque no ápice corneal maior do que $2 \mathrm{~mm}$ para evitar cicatrizes por trauma.

\section{Três pontos de toque}

A finalidade desta técnica é distribuir o peso da LC fazendo um leve toque sobre o ápice do cone e dois apoios nas zonas periféricas. Dessa forma, quatro zonas são criadas e observadas no padrão fluoresceínico: leve toque apical, livramento paracentral, toque na meia-periferia e livramento periférico. $\mathrm{A} \mathrm{CB}$ inicial foi selecionada conforme o meridiano mais plano da córnea ou mais plana nos cones avançados. Para a adaptação da LC, uma vez definida a CB central, avaliou-se a necessidade de abrir curvas periféricas, alterar $\varnothing$ ou usar outros desenhos especiais para ceratocone.

Em todos os casos, o Ø escolhido dependeu do tipo de cone e do desenho da LC. Em cones redondos e centrais, foram utilizados $\varnothing$ pequenos (de 7,5 a 8,5 mm para Monocurva; de 8,8 a 9,2 para Bicurva) e em cones ovais e excêntricos, $\varnothing$ maiores (entre 9,0 e 9,5 mm para Monocurva e entre 9,2 e 9,6 para Bicurva). O $\varnothing$ da LC e o $\varnothing$ da ZO variaram também de acordo com o $\varnothing$ pupilar, a abertura palpebral e a posição da LC.

\section{Orientação e controle da adaptação}

Foi recomendado iniciar a adaptação com quatro horas de uso, aumentando duas horas a cada dois dias até ocupar todo o período de vigília e remover as LC para dormir. Os retornos foram marcados para o final do $1^{\circ}$ mês (entre 30 e 40 dias), final do $3^{\circ}$ mês (entre 90 e 100 dias) e final do $6^{\circ}$ mês (entre 180 e 200 dias). Os pacientes que apresentassem qualquer problema, como desconforto ou perda de LC, deveriam retornar para avaliação sem marcação. Todos receberam treinamento de manuseio e orientações escritas relacionadas à utilização de soluções químicas, aos sinais e sintomas normais e de complicação ocular. O protocolo deste estudo foi aprovado pelo Comitê de Ética do Hospital de Olhos Sadalla Amin Ghanem.

\section{Análise estatística}

As variáveis qualitativas foram representadas por freqüência absoluta (f) e relativa (\%) e as quantitativas, pela média, desvio-padrão, valores mínimo e máximo. A comparação entre os grupos Controle, Monocurva e Soper-McGuire, em relação às suas médias, foi realizada por meio da Análise de Variância a um fator. Quando a suposição de normalidade dos dados foi rejeitada, aplicou-se a prova de Kruskal-Wallis. Para a comparação de proporções, foi utilizado o teste do Qui-quadrado. Os grupos Monocurva e Soper-McGuire foram comparados em relação às variáveis qualitativas pelo teste do Qui-quadrado e, em relação às variáveis quantitativas, com o teste $\mathrm{t}$ de Student para amostras independentes. Quando a suposição de normalidade dos dados foi rejeitada, aplicou-se a prova de Mann-Whitney. A comparação entre esses grupos, em relação ao tempo de permanência com a LC, foi avaliada por meio da curva de sobrevida de Kaplan-Meier e o teste de Wilcoxon. Para todos os testes estatísticos adotou-se um nível de significância de $0,05(\alpha=5 \%)$.

Para o processamento, foi construído um banco de dados com o "software Access" do "Office" 2000. Foram elaborados gráficos e tabelas com o "Word" e o "Excel” do "Office" 2000.

A análise estatística foi realizada com o auxílio dos programas de computador SPSS 10.0, StatXact 4.0, GraphPad InStat 3.05 e GraphPad Prism 3.03. 


\section{RESULTADOS}

A distribuição dos pacientes, conforme dados demográficos e características clínicas antes da adaptação das LC, nos três grupos (Controle, Monocurva externa e Bicurva SoperMcGuire), não apresentou diferenças significativas com relação ao sexo, olho sorteado, idade, idade quando do diagnóstico do ceratocone, Kmin, Kmax, Kmédio, Kmax-Kmin, erro de refração e AV sem correção (Tabela 1).

Quanto à relação LC X córnea pelo padrão fluoresceínico, na avaliação inicial não houve diferença significativa entre os grupos. Em ambos, foi mais freqüente a adaptação da LC com um toque central e dois periféricos, seguida pela com toque apical. Na avaliação final, entretanto, houve diferença significativa ( $p=0,0427$ ) mostrando que no grupo Monocurva ocorreu aumento do porcentual de adaptações com toque apical e no grupo Bicurva Soper-McGuire o aumento foi evidenciado nas adaptações com livramento apical (Tabela 2). Com referência à distribuição do ceratocone, classificado pela localização do ápice, na avaliação inicial não houve diferença significativa na distribuição dos diferentes tipos entre os grupos. Porém, na final observou-se significativa redução significativa do porcentual de cones com ápice central que permaneceram adaptados com LC Monocurvas ( $\mathrm{p}=0,035$ ) (Tabela 3 ).

No que se refere às variações do poder dióptrico da toricidade da córnea (Kmax - Kmin) na avaliação final houve diferença significativa $(\mathrm{p}=0,0249)$, com redução da toricidade corneal tanto com a LC Monocurva quanto com a
Bicurva (Tabela 4). Quanto às variações do poder ceratométrico médio $\{(\mathrm{Kmax}+\mathrm{Kmin}) / 2\}$, houve diferença significativa na avaliação final $(\mathrm{p}=0,0005)$ e na comparação entre as diferenças observadas em cada grupo, nas avaliações inicial e final $(\mathrm{p}=0,0073)$ (Tabela 5).

A AV corrigida com LC, em logMar, sofreu alteração significativa na avaliação final $(\mathrm{p}=0,0037)$, tendo o grupo adaptado com LC Bicurva Soper-McGuire apresentado AV superior (Tabela 6).

Quanto à permanência dos pacientes nos mesmos grupos de estudo, entre os adaptados com LC Monocurva, 57 (90,48\%) participaram da $2^{\underline{q}}$ avaliação, $41(65,08 \%)$ da $3^{\underline{q}}$ avaliação e 35 $(55,56 \%)$ da $4^{\underline{a}}$ avaliação. Entre os adaptados com LC Bicurva Soper-McGuire, $56(88,90 \%)$ compareceram à $2^{\underline{a}}$ visita, 44 $(69,84 \%)$ à $3^{\underline{a}}$ visita e $43(68,25 \%)$ à $4^{\underline{a}}$ visita. As razões pelas quais os pacientes não permaneceram nos mesmos grupos até o final do estudo estão descritas na tabela 7. A estimativa de permanência com o mesmo tipo de LC mostrou que dos 63 pacientes adaptados com LC Monocurva, 38 (60,32\%) completaram o estudo usando o mesmo desenho e dos 63 pacientes adaptados com LC Bicurva, 45 (71,43\%) terminaram o estudo com Bicurva. A diferença entre os grupos não foi estatisticamente significativa $(\mathrm{p}=0,2662)$ (Figura 1$)$.

\section{DISCUSS ÃO}

As primeiras tentativas para adaptar LC no ceratocone são, geralmente, realizadas com as LC Monocurvas, expli-

\begin{tabular}{|c|c|c|c|c|c|}
\hline & $\begin{array}{c}\text { Controle } \\
(n=61)\end{array}$ & $\begin{array}{l}\text { Monocurva } \\
(n=63)\end{array}$ & $\begin{array}{l}\text { Soper-McGuire } \\
\qquad(n=63)\end{array}$ & Teste estatístico & Valor de $p$ \\
\hline Sexo (\%) & & & & Qui quadrado=1,187 GLib=2 & $\mathrm{p}=0,5520$ \\
\hline Masculino & 47,54 & 57,14 & 53,97 & & \\
\hline Feminino & 52,46 & 42,86 & 46,03 & & \\
\hline Olho sorteado (\%) & & & & Qui quadrado $=1,908 \mathrm{GLib}=2$ & $p=0,3850$ \\
\hline Direito & 60,66 & 49,21 & 50,79 & & \\
\hline Esquerdo & 39,34 & 50,79 & 49,21 & & \\
\hline Idade dos sujeitos-anos (média e DP) & $26,00(8,69)$ & $25,17(7,77)$ & $26,56(9,39)$ & ANOVA $(F=0,4071)$ & $p=0,6662$ \\
\hline $\begin{array}{l}\text { Idade quando do diagnóstico-anos } \\
\text { (média e DP) }\end{array}$ & $22,10(8,23)$ & $21,95(7,21)$ & $21,51(6,21)$ & Kruskal-Wallis $\mathrm{KW}=0,03213$ & $p=0,9841$ \\
\hline \multicolumn{6}{|c|}{ Média e desvio padrão da curvatura corneal (dioptrias) } \\
\hline Meridiano horizontal (Kmin) & $49,39(4,40)$ & $47,94(2,46)$ & $48,30(2,91)$ & Kruskal-Wallis $\mathrm{KW}=2,713$ & $p=0,2575$ \\
\hline Meridiano vertical (Kmax) & $52,43(4,21)$ & $52,31(3,25)$ & $53,81(4,92)$ & Kruskal-Wallis $\mathrm{KW}=3,449$ & $p=0,1783$ \\
\hline Toricidade (Kmax - Kmin) & $3,03(4,24)$ & $4,37(2,59)$ & $5,50(3,91)$ & Kruskal-Wallis $\mathrm{KW}=5,527$ & $p=0,0631$ \\
\hline $\mathrm{K}$ médio $(\mathrm{Kmin}+\mathrm{Kmax}) / 2$ & $50,91(3,75)$ & $50,13(2,57)$ & $51,05(3,54)$ & Kruskal-Wallis $\mathrm{KW}=1,939$ & $p=0,3793$ \\
\hline \multicolumn{6}{|l|}{ Média do erro de refração (dioptrias) } \\
\hline Esférico & $-4,58(4,16)$ & $-3,09(4,46)$ & $-4,14(3,79)$ & Kruskal-Wallis $\mathrm{KW}=3,894$ & $p=0,1427$ \\
\hline Cilindro & $-2,69(1,93)$ & $-3,00(1,74)$ & $-2,88(1,95)$ & Kruskal-Wallis $\mathrm{KW}=1,299$ & $p=0,5524$ \\
\hline Equivalente esférico & $-5,92(4,16)$ & $-4,59(4,73)$ & $-5,58(4,00)$ & Kruskal-Wallis $\mathrm{KW}=2,449$ & $p=0,2110$ \\
\hline \multicolumn{6}{|l|}{ Média e desvio padrão da acuidade visual } \\
\hline AV s/correção log(1/fração de Snellen) & $1,37(0,78)$ & $1,17(0,72)$ & $1,41(0,79)$ & Kruskal-Wallis $\mathrm{KW}=3,449$ & $p=0,1783$ \\
\hline
\end{tabular}


332 Avaliação comparativa da adaptação de lentes de contato rígidas gás-permeáveis Monocurvas e Bicurvas (desenho Soper-McGuire) em pacientes portadores de ceratocone: um estudo prospectivo e randomizado

\begin{tabular}{|c|c|c|c|c|c|c|}
\hline \multicolumn{7}{|c|}{$\begin{array}{l}\text { Tabela 2. Relação LC-córnea pelo padrão fluoresceínico nos } \\
\text { grupos Monocurva e Bicurva Soper-McGuire, na } 4^{\circ} \text { avaliação }\end{array}$} \\
\hline \multirow{3}{*}{ 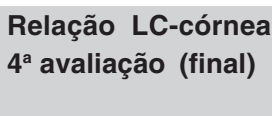 } & \multicolumn{4}{|c|}{ Tipo de LC } & \multirow{2}{*}{\multicolumn{2}{|c|}{ Total }} \\
\hline & \multicolumn{2}{|c|}{ Monocurva } & \multicolumn{2}{|c|}{ Soper-McGuire } & & \\
\hline & $f$ & $\%$ & $f$ & $\%$ & $f$ & $\%$ \\
\hline Livramento apical & 5 & 13,16 & 17 & 37,78 & 22 & 26,51 \\
\hline Toque apical & 12 & 31,58 & 11 & 24,44 & 23 & 27,71 \\
\hline $\begin{array}{l}3 \text { toques: } 1 \text { central } \\
\text { e } 2 \text { periféricos }\end{array}$ & 19 & 50,00 & 17 & 37,78 & 36 & 43,37 \\
\hline Descentralizada & 2 & 5,26 & - & - & 2 & 2,41 \\
\hline Total & 38 & 100,00 & 45 & 100,00 & 83 & 100,00 \\
\hline$\chi^{2}=8,168$ & & $i b=3$ & & 0,0427 & & \\
\hline $\begin{array}{l}\mathrm{LC}=\text { lente } \mathrm{o} \\
\text { graus de lib }\end{array}$ & & $\begin{array}{l}\text { ncia a } \\
\text { dade }\end{array}$ & & $=$ freqüêr & & $\mathrm{G}$ \\
\hline
\end{tabular}

\begin{tabular}{|c|c|c|c|c|c|c|}
\hline \multirow{3}{*}{$\begin{array}{l}\text { Localização } 4^{\mathrm{a}} \\
\text { avaliação (final) }\end{array}$} & \multicolumn{4}{|c|}{ Tipo de LC } & \multirow{2}{*}{\multicolumn{2}{|c|}{ Total }} \\
\hline & \multicolumn{2}{|c|}{ Monocurva } & \multicolumn{2}{|c|}{ Soper-McGuire } & & \\
\hline & $f$ & $\%$ & $f$ & $\%$ & \multicolumn{2}{|r|}{$\%$} \\
\hline Central & 20 & 52,63 & 34 & 75,56 & 54 & 65,06 \\
\hline Paracentral & 15 & 39,47 & 11 & 24,44 & 26 & 31,33 \\
\hline Periférico & 3 & 7,90 & - & - & 3 & 3,61 \\
\hline Total & 38 & 100,00 & 45 & 100,00 & 83 & 100,00 \\
\hline$\chi^{2}=6,702$ & \multicolumn{2}{|c|}{ GLib=2 } & \multicolumn{2}{|c|}{$p=0,035$} & & \\
\hline
\end{tabular}

cando a elevada freqüência de seu uso, principalmente nos cones incipientes e moderados ${ }^{(11,21)}$. Quando não se consegue boa relação LC X córnea com as LC Monocurvas, testam-se as LC Bicurvas tipo Soper, procurando-se obter padrão fluoresceínico com livramento apical ou com toque apical mínimo. Neste estudo, como um dos critérios de inclusão foi pacientes com cones que pudessem ser adaptados tanto com LC Monocurva quanto com LC Bicurva Soper-McGuire, só foram incluídas pessoas cujos cones eram moderados, avançados ou muito avançados.

No presente estudo, a primeira tentativa foi adaptar com livramento apical, mas o exame à lâmpada de fenda mostrou que o padrão fluoresceínico mais freqüente, obtido nos dois grupos, foi o com três toques seguido pelo de toque apical. Nas visitas de acompanhamento foi tentado o livramento apical, mas como 49,2\% dos pacientes eram portadores de ceratocone avançado e severo, este padrão fluoresceínico foi superado pelo de toque apical. O mesmo aconteceu no estudo multicêntrico "CLEK", no qual aproximadamente $88 \%$ dos portadores de ceratocone foram adaptados com LC rígidas fazendo toque apical ${ }^{(22)}$. O padrão fluoresceínico com livramento apical foi, na maioria das vezes, obtido com a utilização de LC de $\varnothing$ pequeno, entre $7,8 \mathrm{~mm}$ e $8,5 \mathrm{~mm}$, e zona óptica ( $\mathrm{ZO}$ ) pequena, entre $2,2 \mathrm{~mm}$ e $2,5 \mathrm{~mm}$ menor que o $\varnothing$, em cones centrais ou paracentrais. Foi necessário, em alguns casos, o uso de $\varnothing$ maiores, devido ao grau e posicionamento do cone, com o objetivo de posicionar a borda superior da LC debaixo da

\begin{tabular}{|c|c|c|c|c|c|c|c|c|}
\hline Meridiano & Tipo de LC & Média & Desvio padrão & Máximo & Mínimo & \multicolumn{2}{|c|}{ Teste estatístico } & \multirow{2}{*}{$\begin{array}{c}\text { Significância } \\
\text { p=0,4525 }\end{array}$} \\
\hline $\begin{array}{l}\text { Kmin } \\
1^{\underline{a}} \text { visita }\end{array}$ & $\begin{array}{r}\text { Mono }(n=63) \\
\text { S-M }(n=63)\end{array}$ & $\begin{array}{l}47,94 \\
48,30\end{array}$ & $\begin{array}{l}2,466 \\
2,91\end{array}$ & $\begin{array}{l}57,31 \\
56,50\end{array}$ & $\begin{array}{l}45,00 \\
41,84\end{array}$ & \multicolumn{2}{|c|}{$t=0,7537 \quad G L=120$} & \\
\hline $\begin{array}{l}\text { Kmin } \\
4^{\underline{a}} \text { visita }\end{array}$ & $\begin{array}{r}\text { Mono }(n=38) \\
\text { S-M }(n=45)\end{array}$ & $\begin{array}{l}46,98 \\
48,40\end{array}$ & $\begin{array}{l}2,27 \\
3,46\end{array}$ & $\begin{array}{l}54,52 \\
56,75\end{array}$ & $\begin{array}{l}43,40 \\
39,44\end{array}$ & $t=2,248$ & $\mathrm{GL}=76$ & $p=0,0275$ \\
\hline $\begin{array}{l}\text { Kmin } \\
\text { Diferenças }\end{array}$ & $\begin{array}{r}\text { Mono }(n=38) \\
\text { S-M }(n=45)\end{array}$ & $\begin{array}{r}0,59 \\
-0,34\end{array}$ & $\begin{array}{l}1,12 \\
2,57\end{array}$ & $\begin{array}{l}2,84 \\
6,06\end{array}$ & $\begin{array}{l}-1,87 \\
-7,60\end{array}$ & $t=2,187$ & $\mathrm{GL}=62$ & $p=0,0325$ \\
\hline $\begin{array}{l}\text { Completaram } \\
\text { Não completaram }\end{array}$ & $\begin{array}{l}\text { Mono }(n=38) \\
\text { Mono }(n=25)\end{array}$ & $\begin{array}{l}47,57 \\
48,51\end{array}$ & $\begin{array}{l}2,11 \\
2,86\end{array}$ & $\begin{array}{l}53,28 \\
57,31\end{array}$ & $\begin{array}{l}45,00 \\
45,43\end{array}$ & $t=1,403$ & $\mathrm{GL}=40$ & $p=0,1685$ \\
\hline $\begin{array}{l}\text { Completaram } \\
\text { Não completaram }\end{array}$ & $\begin{array}{l}\text { S-M }(n=45) \\
\text { S-M }(n=18)\end{array}$ & $\begin{array}{l}48,07 \\
48,90\end{array}$ & $\begin{array}{l}2,93 \\
2,83\end{array}$ & $\begin{array}{l}56,50 \\
54,41\end{array}$ & $\begin{array}{l}41,84 \\
45,10\end{array}$ & $t=1,046$ & $\mathrm{GL}=32$ & $p=0,3035$ \\
\hline
\end{tabular}

\begin{tabular}{|c|c|c|c|c|c|c|c|}
\hline Meridiano & Tipo de LC & Média & Desvio padrão & Máximo & Mínimo & Teste estatístico & Significância \\
\hline $\begin{array}{l}\text { Kmax } \\
1^{\underline{a}} \text { visita }\end{array}$ & $\begin{array}{r}\text { Mono }(n=63) \\
\text { S-M }(n=63)\end{array}$ & $\begin{array}{l}52,31 \\
53,87\end{array}$ & $\begin{array}{l}3,25 \\
4,92\end{array}$ & $\begin{array}{l}60,62 \\
68,21\end{array}$ & $\begin{array}{l}46,83 \\
45,75\end{array}$ & $\mathrm{GL}=107$ & $p=0,0566$ \\
\hline $\begin{array}{l}\text { Kmax } \\
4^{\underline{a}} \text { visita }\end{array}$ & $\begin{array}{r}\text { Mono }(n=38) \\
\text { S-M }(n=45)\end{array}$ & $\begin{array}{l}50,76 \\
53,73\end{array}$ & $\begin{array}{l}2,55 \\
4,17\end{array}$ & $\begin{array}{l}56,63 \\
64,33\end{array}$ & $\begin{array}{l}46,41 \\
46,25\end{array}$ & $\begin{array}{c}\text { Mann-Whitney } \\
\text { U }=461,5\end{array}$ & $p=0,0003$ \\
\hline $\begin{array}{l}\text { Kmax } \\
\text { Diferenças }\end{array}$ & $\begin{array}{r}\text { Mono }(n=38) \\
\text { S-M }(n=45)\end{array}$ & $\begin{array}{l}1,16 \\
0,04\end{array}$ & $\begin{array}{l}1,73 \\
2,62\end{array}$ & $\begin{array}{l}4,83 \\
6,21\end{array}$ & $\begin{array}{l}-2,69 \\
-7,75\end{array}$ & $\mathrm{t}=2,334 \quad \mathrm{GL}=76$ & $p=0,0222$ \\
\hline $\begin{array}{l}\text { Completaram } \\
\text { Não completaram }\end{array}$ & $\begin{array}{l}\text { Mono }(n=38) \\
\text { Mono }(n=25)\end{array}$ & $\begin{array}{l}51,92 \\
59,91\end{array}$ & $\begin{array}{l}3,14 \\
3,38\end{array}$ & $\begin{array}{l}59,28 \\
60,62\end{array}$ & $\begin{array}{l}46,83 \\
47,92\end{array}$ & $t=1,171$ & $\mathrm{p}=0,2473$ \\
\hline $\begin{array}{l}\text { Completaram } \\
\text { Não completaram }\end{array}$ & $\begin{array}{l}\text { S-M }(n=45) \\
\text { S-M }(n=18)\end{array}$ & $\begin{array}{l}53,77 \\
53,91\end{array}$ & $\begin{array}{l}4,85 \\
5,25\end{array}$ & $\begin{array}{l}68,21 \\
64,50\end{array}$ & $\begin{array}{l}45,75 \\
46,75\end{array}$ & $t=0,09712$ & $p=0,9233$ \\
\hline
\end{tabular}




\begin{tabular}{|c|c|c|c|c|c|c|c|}
\hline Acuidade visual & Tipo de LC & Média & Desvio padrão & Máximo & Mínimo & Teste estatístico & Significância \\
\hline $1^{a}$ visita & $\begin{array}{r}\text { Mono }(n=63) \\
\text { S-M }(n=63)\end{array}$ & $\begin{array}{l}0,21 \\
0,24\end{array}$ & $\begin{array}{l}0,14 \\
0,13\end{array}$ & $\begin{array}{l}0,90 \\
0,60\end{array}$ & $\begin{array}{l}0,00 \\
0,00\end{array}$ & $\begin{array}{c}\text { Mann-Whitney } \\
U=1645,0\end{array}$ & $p=0,0965$ \\
\hline $4^{\mathrm{a}}$ visita (final) & $\begin{array}{r}\text { Mono }(n=38) \\
\text { S-M }(n=45)\end{array}$ & $\begin{array}{l}0,20 \\
0,24\end{array}$ & $\begin{array}{l}0,16 \\
0,11\end{array}$ & $\begin{array}{l}0,90 \\
0,60\end{array}$ & $\begin{array}{l}0,10 \\
0,00\end{array}$ & $\begin{array}{c}\text { Mann-Whitney } \\
U=538,5\end{array}$ & $p=0,0037$ \\
\hline Diferenças & $\begin{array}{r}\text { Mono }(n=38) \\
\text { S-M }(n=45)\end{array}$ & $\begin{array}{l}0,01 \\
0,00\end{array}$ & $\begin{array}{l}0,05 \\
0,09\end{array}$ & $\begin{array}{l}0,20 \\
0,20\end{array}$ & $\begin{array}{l}-0,10 \\
-0,30\end{array}$ & $\begin{array}{c}\text { Mann-Whitney } \\
\text { U }=804,0\end{array}$ & $p=0,6378$ \\
\hline $\begin{array}{l}\text { Completaram } \\
\text { Não completaram }\end{array}$ & $\begin{array}{l}\text { Mono }(n=38) \\
\text { Mono }(n=25)\end{array}$ & $\begin{array}{l}0,20 \\
0,22\end{array}$ & $\begin{array}{l}0,16 \\
0,09\end{array}$ & $\begin{array}{l}0,90 \\
0,50\end{array}$ & $\begin{array}{l}0,00 \\
0,10\end{array}$ & $\begin{array}{c}\text { Mann-Whitney } \\
\text { U }=370,5\end{array}$ & $p=0,1420$ \\
\hline $\begin{array}{l}\text { Completaram } \\
\text { Não completaram }\end{array}$ & $\begin{array}{l}\text { S-M }(n=45) \\
\text { S-M }(n=18)\end{array}$ & $\begin{array}{l}0,24 \\
0,25\end{array}$ & $\begin{array}{l}0,12 \\
0,15\end{array}$ & $\begin{array}{l}0,60 \\
0,50\end{array}$ & $\begin{array}{l}0,10 \\
0,00\end{array}$ & $\begin{array}{c}\text { Mann-Whitney } \\
\text { U }=399,0\end{array}$ & $p=0,9331$ \\
\hline
\end{tabular}

\begin{tabular}{|c|c|c|c|c|c|c|}
\hline \multirow[t]{2}{*}{ Razões } & \multicolumn{2}{|c|}{$2^{\text {a }}$ avaliação } & \multicolumn{2}{|c|}{$3^{\text {a }}$ avaliação } & \multicolumn{2}{|c|}{ 4ª avaliação } \\
\hline & $f$ & $\%$ & $f$ & $\%$ & $f$ & $\%$ \\
\hline \multicolumn{7}{|c|}{ Troca da LC Monocurva por: } \\
\hline LC Soper-McGuire & 4 & 16,00 & 6 & 24,00 & 2 & 8,00 \\
\hline LCG Monocurva & 1 & 4,00 & - & - & - & - \\
\hline LCG tórica & - & - & 1 & 4,00 & 1 & 4,00 \\
\hline LCG Harrison & - & - & 1 & 4,00 & 1 & 4,00 \\
\hline LCG tricurve & - & - & - & - & 1 & 4,00 \\
\hline Sistema "piggyback" & - & - & 2 & 8,00 & 1 & 4,00 \\
\hline Desistência das LC & 1 & 4,00 & 2 & 8,00 & 1 & 4,00 \\
\hline Total & 6 & 24,00 & 12 & 48,00 & 7 & 28,00 \\
\hline \multicolumn{7}{|c|}{ Troca LC Soper-McGuire por: } \\
\hline LC Monocurva & 3 & 16,67 & 3 & 16,67 & - & - \\
\hline LCG tórica & - & - & 2 & 11,11 & - & - \\
\hline LCG tricurve & - & - & 2 & 11,11 & - & - \\
\hline Softperm & - & - & - & - & 2 & 11,11 \\
\hline Sistema "piggyback" & - & - & - & - & 2 & 11,11 \\
\hline Transplante de córnea & - & - & 1 & 5,56 & - & - \\
\hline Desistência das LC & - & - & 2 & 11,11 & 1 & 5,56 \\
\hline Total & 3 & 16,67 & 10 & 55,56 & 5 & 27,78 \\
\hline
\end{tabular}

pálpebra superior, prevenindo seu deslocamento durante o piscar. Quando não se conseguiu a adaptação com livramento apical, buscou-se a adaptação com três pontos de toque. Um discreto toque apical regularizou mais a superfície anterior da córnea proporcionando AV melhor para alguns pacientes.

A técnica de toque apical é considerada um método prático e simples desde a década de $50^{(23)}$. Apesar de proporcionar AV mais nítida, pela diminuição da irregularidade da córnea, alguns estudos mostraram que esta técnica provoca e acelera a formação de cicatrizes corneais, pelo trauma crônico ${ }^{(24-25)}$. Foi demonstrado que LC RGP planas, com $\varnothing 9,4 \mathrm{~mm}$, produziram mudanças no ápice e cicatrizes, enquanto a utilização de LC do mesmo material, com $\varnothing 8,0 \mathrm{~mm}$, adaptadas com livramento apical e usadas durante o mesmo período não causaram alterações apicais ${ }^{(25)}$. As cicatrizes corneais e o astigmatismo irregular diminuem significativamente a $\mathrm{AV}$, especialmente a de baixo contraste ${ }^{(26)}$. Publicação do grupo "CLEK" sugeriu que o desenvolvimento de cicatrizes corneais no ceratocone esteja associado à ceratopatia superficial, uso de LC, presença de anel de Fleischer, altos valores ceratométricos e aumento da idade $^{(24)}$. Estudo recente deste mesmo grupo, comparando a adaptação com toque apical e livramento apical, não encontrou diferença significativa no que se refere à formação de cicatrizes, depois de 8 anos de seguimento ${ }^{(27)}$. No presente estudo, sempre que possível, evitou-se a adaptação com toque apical. Apesar disso, 31,75\% das LC Monocurvas e 28,57\% das LC Soper-McGuire apresentaram toque apical na $1^{\underline{a}}$ avaliação, tendo o grupo LC Monocurva mostrado redução significativa do padrão de livramento apical na última avaliação. No grupo LC Soper-McGuire também houve predominância do padrão de adaptação com três toques, mas foi seguido pelo de livramento apical a partir da $2^{\underline{q}}$ visita (Tabela 2 ). A adaptação com o desenho tipo Soper visa principalmente evitar ou minimizar o toque apical. 


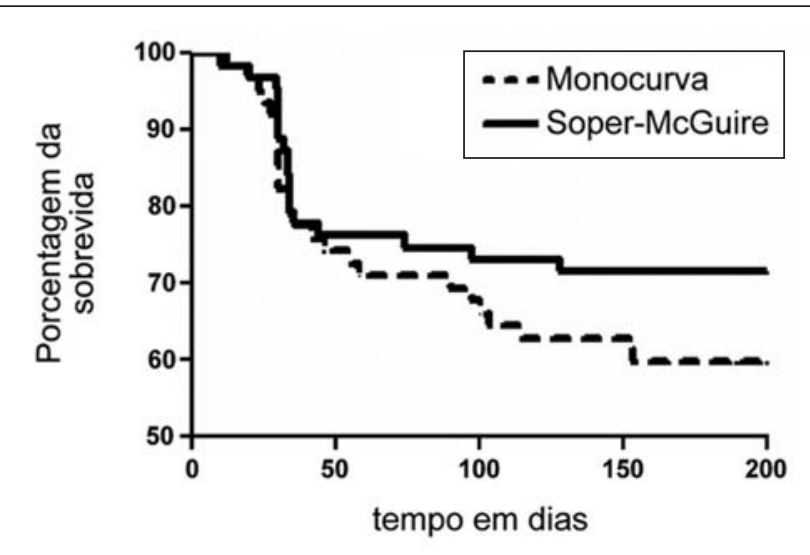

Comparação da sobrevivência usando o teste estatístico de Wilcoxon (Gehan)=1,236 G Lib=1 $p=1,2662$

Figura 1 - Curvas de sobrevivência de Kaplan-Meier mostrando a estimativa de permanência no uso das LC Monocurva ou Bicurva Soper-Mcguire

As alterações na distribuição dos diferentes padrões da relação LC X córnea, observadas nos dois grupos, podem refletir mudanças topográficas da córnea relacionadas com a evolução do cone, com a filosofia de adaptação das LC ou, simplesmente, serem conseqüências do uso contínuo das mesmas, já que estudos comprovaram que no ceratocone a resistência do tecido corneal e a rigidez escleral são mais baixas que o normal ${ }^{(28-30)}$. Outras causas para as variações do padrão fluoresceínico, encontradas entre a $1^{\underline{a}}$ visita e o exame final, podem ter sido a dificuldade de reprodutibilidade do padrão fluoresceínico pela variedade da concentração e quantidade de fluoresceína instilada; as mudanças químicas e físicas da lágrima (osmolaridade, $\mathrm{pH}$, viscosidade, concentração protéica); a movimentação e o posicionamento da LC; e as forças externas relacionadas à compressão, ao posicionamento e à mobilidade das pálpebras ${ }^{(31-35)}$.

Quanto à distribuição dos diferentes tipos de cone, classificados pela localização do ápice, na última visita, o grupo de portadores de cone central adaptado com LC Monocurva reduziu significativamente $(\mathrm{p}=0,035)$ (Tabela 3$)$. Observouse, também na avaliação final, que a redução do número de cones centrais teve em contrapartida um aumento de cones paracentrais. Este fato pode ter ocorrido pelo efeito mecânico da LC sobre a córnea ou pela evolução do cone. Estudos demonstraram que à medida que o ceratocone se desenvolve, o ápice corneal é usualmente deslocado para a região inferior $^{(36-37)}$. Alterações que ocorrem em pacientes portadores de ceratocone, usuários de LC RGP, podem refletir um efeito mecânico da LC na córnea, mas nem sempre esse efeito é claro, pois há limitação na obtenção de dados topográficos nos olhos com estágio avançado da doença ${ }^{(38)}$.

Neste estudo, a LC Monocurva determinou aplanamento do Kmin e do Kmax e a LC Soper-McGuire provocou encurvamento do Kmin e aplanamento do Kmax (Tabelas 4 e 5).
Embora existam relatos que a causa da deformação da córnea pela LC relaciona-se principalmente com alterações fisiológicas associadas com longo tempo de hipóxia ${ }^{(39-40)}$, o material das LC usadas neste estudo tinham Dk/L suficiente para evitar hipóxia, além de terem sido removidas para dormir. As LC RGP são capazes de induzir mecanicamente alterações na topografia corneal de olhos $\operatorname{sadios}^{(30,41-42)}$ e muito mais nos olhos portadores de ceratocone ${ }^{(28-29)}$. Por isso, as alterações topográficas observadas, embora possam refletir mudanças de curvatura da córnea relacionadas com a evolução do cone, considerando-se o período de acompanhamento dos pacientes podem ser creditadas, na maior parte, ao uso das LC.

No que diz respeito à $\mathrm{AV}$, foi publicado que pacientes adaptados com toque apical apresentaram melhor AV pelo aplanamento do cone ${ }^{(19,43)}$. Entretanto, os resultados deste estudo, após seis meses de seguimento, mostraram redução de AV nos adaptados com LC Monocurvas, que fizeram mais toque apical, em relação aos adaptados com LC SoperMcGuire ( $\mathrm{p}=0,0037$ ) (Tabela 6).

As LC rígidas continuam sendo a principal forma de correção óptica do ceratocone, pois a AV alcançada é geralmente melhor ou igual a $20 / 40^{(2,11,19)}$. Além disso, reduzem as aberrações ópticas e melhoram a sensibilidade ao contras$\mathrm{te}^{(44)}$, o que permite aos pacientes manterem suas atividades normais.

Quanto à estimativa de permanência no uso do mesmo tipo de LC, no grupo adaptado com Monocurva foi de $60,32 \%$ e no grupo adaptado com Bicurva foi de $71,43 \%$. A diferença entre os grupos não foi estatisticamente significativa $(\mathrm{p}=0,2662)$ (Figura 1), mas clinicamente importante. Os pacientes adaptados com LC Soper-McGuire apresentaram $11,11 \%$ de probabilidade a mais que os do grupo LC Monocurva de, ao final do estudo, continuarem adaptados com LC do mesmo desenho, demonstrando tendência a favor das lentes Bicurvas para cones avançados e muito avançados. Nos graus avançado e muito avançado, onde o toque central foi geralmente mais acentuado com as LC Monocurvas, elas foram mudadas para LC Soper-McGuire. Alguns pacientes com ceratocone central avançado desenvolveram nódulo fibroblástico na área superficial da córnea. O trauma causado pela LC rígida sobre o nódulo provocou erosão no ápice do cone ou bem próximo dele. A suspensão da LC permitiu a reepitelização do nódulo, mas mesmo com a readaptação de uma LC rígida com maior livramento apical, ocorreu nova erosão, tornando muito desconfortável o seu uso. Para protelar a cirurgia, esses pacientes foram readaptados com LC gelatinosas especiais ou com o sistema de adaptação à cavaleiro ("piggyback"), uma LC RGP sobre uma LCG. Com o advento das LC RGP de alto Dk, das LCG com alto conteúdo aquoso e, mais recentemente, das LC de silicone-hidrogel, esse sistema de adaptação passou a ser utilizado com maior freqüência, retardando ou evitando o transplante de córnea ${ }^{(11,45)}$ (Tabela 7). No estudo de Ghanem et al. ${ }^{(11)}$, em 2003, o sistema à cavaleiro foi mais adaptado nos cones centrais 
com erosão recorrente de ápice, para aliviar o desconforto e permitir a reepitelização sem a suspensão do uso de LC.

Alguns casos deste estudo foram readaptados com LCG. As LCG com desenho especial para ceratocone, cujo centro é menos flexível que a periferia, podem fornecer visão aceitável e conforto para pacientes com cones de $\emptyset$ grande e com localização central ou paracentral. As LCG tóricas são mais bem adaptadas nos cones centrais, possivelmente por ter a córnea com esse tipo de cone uma configuração mais semelhante ao desenho padrão da LC, facilitando o posicionamento e a estabilidade do eixo ${ }^{(11)}$.

Este estudo mostra que devido às variações acentuadas na topografia corneal das pessoas portadoras de ceratocone, um tipo único de LC não proporciona adaptação ideal para todos os casos e que esses pacientes devem ser examinados a cada seis ou 12 meses, a fim de que essas mudanças sejam detectadas e as LC readaptadas para evitar trauma e formação de cicatrizes no ápice corneal.

A homogeneidade dos dois grupos assegurou sua validade interna no que diz respeito às possíveis influências dos diferentes tipos de LC, nas variáveis clínicas analisadas. Importante, também, foi comparar os dados demográficos e os aspectos clínicos analisados dos 61 pacientes do grupo controle. Esses indivíduos não apresentaram diferenças significativas quando comparados com os pacientes incluídos no estudo. Portanto, confirmou a validade externa dos resultados desta investigação para os 210 pacientes, portadores de ceratocone com indicação para o uso de LC, inicialmente avaliados.

\section{CONCLUSÕES}

$\mathrm{O}$ estudo mostrou que devido às variações observadas na topografia corneal, o mesmo desenho de LC usado no início da adaptação não proporcionou a melhor relação lente $\mathrm{X}$ córnea com o passar do tempo. A lente Soper-McGuire demonstrou melhor desempenho clínico do que a Monocurva em ceratocones avançados e centrais.

\section{ABSTRACT}

Purpose: To evaluate the clinical performance of Monocurve and Bicurve (Soper-McGuire design) rigid gas-permeable contact lens fitting in patients with keratoconus. Methods: A prospective and randomized comparative clinical trial was conducted with a minimum follow-up of six months in two groups of 63 patients. One group was fitted with Monocurve contact lenses and the other with Bicurve Soper-McGuire design. Study variables included fluoresceinic pattern of lens-to-cornea fitting relationship, location and morphology of the cone, presence and degree of punctate keratitis and other corneal surface alterations, topographic changes, visual acuity for distance corrected with contact lenses and survival analysis for remaining with the same contact lens design during the study. Results: During the follow-up there was a decrease in the number of eyes with advanced and central cones fitted with Monocurve lenses, and an increase in those fitted with Soper-McGuire design. In the Monocurve group, a flattening of both the steepest and the flattest keratometric curve was observed. In the SoperMcGuire group, a steepening of the flattest keratometric curve and a flattening of the steepest keratometric curve were observed. There was a decrease in best-corrected visual acuity with contact lens in the Monocurve group. Survival analysis for the Monocurve lens was $60.32 \%$ and for the Soper-McGuire was $71.43 \%$ at a mean follow-up of six months. Conclusions: This study showed that due to the changes observed in corneal topography, the same contact lens design did not provide an ideal fitting for all patients during the follow-up period. The Soper-McGuire lenses had a better performance than the Monocurve lenses in advanced and central keratoconus.

Keywords: Keratoconus; Contact lenses; Clinical evolution; Corneal topography; Visual acuity

\section{REFERÊNCIAS}

1. Krachmer JH, Feder RS, Belin MW. Keratoconus and related noninflammatory corneal thinning disorders. Surv Ophthalmol. 1984;28(4):293-322.

2. Rabinowitz YS. Keratoconus. Surv Ophthalmol. 1998;42(4):297-319. Review.

3. Griffiths M, Zahner K, Collins M, Carney L. Masking of irregular corneal topography with contact lenses. CLAO J. 1998;24(2):76-81.

4. Buxton JN. Contact lenses in keratoconus. Contact Intraocul Lens Med J. 1978;4:74-85

5. Carney LG. Contact lens correction of visual loss in keratoconus. Acta Ophthalmol (Copenh). 1982;60(5):795-802.

6. Buxton JN, Keattes RH, Hoeffle FB, et al. The contact lens correction of keratoconus. In: Dabezies OH Jr. The CLAO guide to basic science and clinical practice. Orlando: Grune \& Stratton; 1984. p.55:1-55.14.

7. Belin MW. Optical and surgical correction of keratoconus - Focal points: 1988: Clinical Module for Ophthalmologists. San Francisco: American Academy of Ophthalmology; 1988.

8. Kastl PR, Donzis PB, Cole HP $3^{\text {rd }}$, Rice J, Baldone JA. A 20-year retrospective study of the use of contact lenses in keratoconus. CLAO J. 1987;13(2): 102-4.

9. Smiddy WE, Hamburg TR, Kracher GP, Stark WJ. Keratoconus. Contact lens or keratoplasty? Ophthalmology. 1988;95(4):487-92.

10. Leça RG, Fukushima N, Gonzaga R, Lipener C, Zamboni F, Lewinsky R, Uras R. Estudo clínico da adaptação de lentes de contato no ceratocone. Arq Bras Oftalmol. 1995;58(3):149-51.

11. Ghanem VC, Coral-Ghanem C, Ghanem RC, Larinho C. Ceratocone: correlação entre grau evolutivo e padrão topográfico com o tipo de lente de contato adaptada. Arq Bras Oftalmol. 2003;66(2):129-35.

12. Garcia-Lledo M, Feinbaum C, Alio JL. Contact lens fitting in keratoconus. Compr Ophthalmol Update. 2006;7(2):47-52.

13. Lass JH, Lembach RG, Park SB, Hom DL, Fritz ME, Svilar GM, et al. Clinical management of keratoconus. A multicenter analysis. Ophthalmology. 1990;97(4):433-45.

14. Benett ES. Keratoconus. In: Bennett ES, Grohe RM, editors. Rigid gaspermeable contact lenses. New York: Professional Press Books; 1986. p.297-344.

15. Dana MR, Putz JL, Viana MA, Sugar J, McMahon TT. Contact lens failure in keratoconus management. Ophthalmology. 1992;99(8):1187-92. Comment in: Ophthalmology. 1992;99(12):1752-3. Ophthalmology. 1993;100(2):147-8.

16. Lim N, Vogt U. Characteristics and functional outcomes of 130 patients with keratoconus attending a specialist contact lens clinic. Eye. 2002;16(1):54-9. 
336 Avaliação comparativa da adaptação de lentes de contato rígidas gás-permeáveis Monocurvas e Bicurvas (desenho Soper-McGuire) em pacientes portadores de ceratocone: um estudo prospectivo e randomizado

17. Mannis MJ, Zadnik K. Contact lens fitting in keratoconus. CLAO J. 1989; 15(4):282-9. Review.

18. Mandell RB. Contemporary management of keratoconus. Int Contact Lens Clin. 1997;24(2):43-58.

19. Lee JL, Kim MK. Clinical performance and fitting characteristics with a multicurve lens for keratoconus. Eye Contact Lens. 2004;30(1):20-4.

20. Soper JW, Jarrett A. Results of a systematic approach to fitting keratoconus and corneal transplants. Contact Lens Med Bull. 1972;5:50-9.

21. Crews MJ, Driebe WT Jr, Stern GA. The clinical management of keratoconus: a 6 year retrospective study. CLAO J. 1994;20(3):194-7.

22. Szczotka LB, Barr JT, Zadnik K. A summary of the findings from the Collaborative Longitudinal Evaluation of Keratoconus (CLEK) Study. CLEK Study Group. Optometry. 2001;72(9):574-84.

23. Chiquiar Arias V, Liberatore JC, Voss EH, Chiquiar Arias M. A new technique of fitting contact lenses on keratoconus. Contacto. 1959;3:393-415.

24. Barr JT, Zadnik K, Wilson BS, Edrington TB, Everett DF, Fink BA, et al. Factors associated with corneal scarring in the Collaborative Longitudinal Evaluation of Keratoconus (CLEK) Study. Cornea. 2000;19(4):501-7.

25. Korb DR, Finnemore VM, Herman JP. Apical changes and scarring in keratoconus as related to contact lens fitting techniques. J Am Optom Assoc. 1982;53(3):199-205.

26. Barr JT, Yackels T. Corneal scarring in keratoconus - measurements and influence on visual acuity. Int Contact Lens Clin. 1991;22(7):173-5.

27. Zadnik K, Barr JT, Steger-May K, Edrington TB, McMahon TT, Gordon MO; The Collaborative Longitudinal Evaluation of Keratoconus (CLEK) Study Group. Comparison of flat and steep rigid contact lens fitting methods in keratoconus. Optom Vis Sci. 2005;82(12):1014-21.

28. Hartstein J, Becker B. Research into the pathogenesis of keratoconus. A new syndrome: low ocular rigidity, contact lenses, and keratoconus. Arch Ophthalmol. 1970;84(6):728-9.

29. Edmund C. Corneal elasticity and ocular rigidity in normal and keratoconic eyes. Acta Ophthalmol (Copenh). 1988;66(2):134-40.

30. Moon JW, Shin KC, Lee HJ, Wee WR, Lee JH, Kim MK. The effect of contact lens wear on the ocular surface changes in keratoconus. Eye Contact Lens. 2006;32(2):96-101.
31. Romanchuk KG. Fluorescein. Physiochemical factors affecting its fluorescence. Surv Ophthalmol. 1982;26(5):269-83.

32. Knoll HA, Conway HD. Analysis of blink-induced vertical motion of contact lenses. Am J Optom Physiol Opt. 1987;64(2):153-5.

33. Mandell RB. Fitting methods and philosophies. In: Mandell RB. Contact lens practice. Springfield, IL: Thomas; 1988. p.226-43.

34. Craig EL. Fluorescein and other dues. In: Mauger TF, Craig EL, editors. Havener's ocular pharmalogogy. $6^{\underline{a}}$ ed. St Louis: Mosby; 1994. p.451-67

35. Fink BA, Barr JT, Edrington TB, Pierce GE, Schechtman KB, Rah MJ, Flom R, Zadnik K; CLEK Study Group. Collaborative Longitudinal Evaluation of Keratoconus. A comparison of two methods of evaluating corneato-contact lens base curve fluorescein patterns in keratoconus. Optom Vis Sci. 2001;78(8):589-98.

36. Maguire LJ, Bourne WM. Corneal topography of early keratoconus. Am J Ophthalmol. 1989;108(2):107-12.

37. Wilson SE, Lin DT, Klyce SD. Corneal topography of keratoconus. Cornea. 1991;10(1):2-8

38. Szczotka LB, Rabinowitz YS, Yang H. Influence of contact lens wear on the corneal topography of keratoconus. CLAO J. 1996;22(4):270-3.

39. Carney LG. Corneal topography changes during contact lens wear. Contact Lens J. 1974;3(1):5-16.

40. Carney LG. The basis for corneal shape change during contact lens wear. Am J Optom Physiol Opt. 1975;52(7):445-54.

41. Wilson SE, Lin DT, Klyce SD, Reidy JJ, Insler MS. Topographic changes in contact lens-induced corneal warpage. Ophthalmology. 1990;97(6):734-44.

42. Maeda N, Klyce SD, Hamano H. Alteration of corneal asphericity in rigid gas permeable contact lens induced warpage. CLAO J. 1994;20(1):27-31.

43. Zadnik K, Mutti DO. Contact lens fitting relation and visual acuity in keratoconus. Am J Optom Physiol Opt. 1987;64(9):698-702.

44. Xie PY, Wang D, Yang LN, Zhou WJ. [The evaluation of visual quality in keratoconus eyes corrected by rigid gas-permeable contact lens]. Zhonghua Yan Ke Za Zhi. 2005;41(12):1086-91. Chineese.

45. Jaworski P, Wygledowska-Promienska D, Gierek-Ciaciura S. [Application of duo-systems (piggy back) in correction of keratoconus]. Klin Oczna. 2004; 106(4-5):629-32. Polish. 